\title{
Judgments of short time intervals following awakenings from different EEG stages of sleep
}

\author{
V. R. CARLSON \\ National Institute of Mental Health, Bethesda, Maryland 20014
}

and

\section{FEINBERG}

Veterans Administration Hospital and University of California at San Francisco San Francisco, California 94121

\begin{abstract}
Twenty subjects were awakened from EEG sleep stages 1-REM, 2, or 4 and tested on estimation, production, and reproduction of time intervals from 1 to 9 secs. A comparison group of 20 subjects was tested according to a similar schedule during the day, and oral temperature readings were obtained at the day sessions. All subjects were young male adults. Subjective time rate decreased to a minimum somewhat past the middle of the sleep period and increased in the morning with little change during the day and no differential association with EEG stage. A tendency toward indiscriminate responding was identified which increased more markedly during the night than the change in time rate and exhibited a minimum near noon. A constant response error was greater following awakenings from stage 2 than from stage 1-REM. Subjective time rate appears to follow a circadian variation which generally parallels the sleep and body temperature cycles but is not specifically dependent on body temperature or the particular EEG stage of sleep.
\end{abstract}

Time judgments made by subjects awakened from sleep may indicate a slowed subjective time sense (Aschoff, Giedke, Poppel, \& Wever, 1972; Wever, 1972). This result would be consistent with the slowing of an "internal clock" dependent upon lowered metabolic activity in the periphery, since body temperature, oxygen consumption, and related variables also show a decrease during the night (Snyder \& Scott, 1972). While metabolic activity in the brain probably differs in its characteristics between sleep and waking, there is no evidence that its overall rate is reduced during sleep (Mangold, Sokoloff, Conner, Kleinerman, Therman, \& Kety, 1955; Reivich, Isaacs, Evarts, \& Kety, 1967).

Time judgments made upon awakening from sleep might also differ from those obtained during normal waking hours as a result of factors other than a change in subjective time rate. Subjects awakened from sleep may be more confused or less motivated to perform the task well, tending to respond more randomly than normal. In terms of an internal clock, subjective rate is reflected in the slope of the function relating the subject's estimates to the actual values of a set of differing time intervals presented for judgment. Random responding would result in a decrease in the slope and an increase in the intercept of the function. An extreme instance of this

Address requests for reprints to: V. R. Carlson, Laboratory of Psychology and Psychopathology, National Institute of Mental Health, Building 10, Room 2N246, Bethesda, Maryland 20014. kind of effect was obtained in a group of schizophrenic subjects, whose (daytime) response functions showed zero slope with intercepts equal to the mean responses (Carlson \& Feinberg, 1968). The individual mean responses were reliable, but these subjects failed to discriminate among the presented time intervals.

A tendency toward indiscriminate responding is distinguishable from a change in subjective time rate by a consideration of the response $\times$ time functions for both time estimation and time production. In the method of estimation, the experimenter presents time intervals and the subject verbally estimates their durations. In the method of production, the experimenter states the lengths of the intervals and the subject tries to "produce" these durations by holding down a key for the stated lengths of time. The method of reproduction combines operations of estimation and production, the experimenter presenting an actual time interval on each trial for the subject to produce. If the subjects' responses are discriminating and consistent for all three methods, the estimation and production functions will be reciprocally related and the slope of the reproduction function will be equal to 1 . Randomness of response with respect to the given time intervals will tend to decrease the slope and equalize the intercepts and means for all three functions, attenuating the reciprocal relationship between estimation and production. Mean responses or responses to a single given interval might increase, 
decrease, or remain unchanged. ${ }^{1}$

A difference in time judgment during the night may be associated either with the particular EEG sleep stage preceding awakening or with the longerterm changes in the metabolic and physiological functions occurring over the entire night. If the difference is due to decreased cognitive or perceptual competence associated with depth of sleep, one might expect a quick decline to a minimum early in the night followed by a gradual return to the daytime level of performance; if the change is associated primarily with lessened metabolic rate, one would expect a more gradual decline to a minimum later in the night (Snyder \& Scott, 1972).

The purpose of the present investigation was to examine the changes that occur over the night in the slopes and intercepts of time judgment functions to see whether these changes are consistent among the several time judgment methods and to determine whether the changes are related to the EEG stage of sleep prior to making the judgments.

\section{METHOD}

\section{Subjects}

Twenty male paid volunteer subjects $18-24$ years of age, were tested over the course of one night's sleep. Twenty other male subjects, also 18-24 years old, were tested during the day according to a schedule similar to that for the Night subjects. The Day subjects were students on summer appointments as assistants in various laboratories. They resumed their normal workday activities between testing sessions. All subjects were tested individually.

\section{EEG Recording}

For the Night subjects, two EEG and two eye-movement electrodes were attached for sleep recording. Electrode placement and criteria for identifying sleep stages were as described in Feinberg, Koresko, and Heller (1967).

\section{Time Judgment Procedure and Apparatus}

On each trial, the subject was informed of whether that trial would be an estimation, production, or reproduction trial. For production, he was also informed of the number of seconds of the time interval to be produced. On every trial, the subject started the trial by pressing a response switch, which turned on a stimulus light. On estimation and reproduction trials, the light was turned off by an interval timer, and the subject released the switch. For estimation, he stated his estimate in seconds of the time the light had been on. For production, he released the switch, which turned off the light, when he thought the required interval of time had elapsed. On a reproduction trial, he pressed the switch a second time, turning the light on again, and released the switch, extinguishing the light, when he judged the second interval of light-on to be equal to the first.

The three kinds of trial were thus as similar as possible with respect to the mechanics of the task. The subject's verbal estimate was recorded for estimation; the actual time (to the nearest $.01 \mathrm{sec}$ ) of holding the switch closed was recorded for production and reproduction. The apparatus was constructed to indicate switch closures or releases made at inappropriate times, and time intervals for which mistrials occurred were repeated later in the same test session.

The response switch was a hand-held microswitch with detent action providing a slight click and a positive "feel" for on and off operation. The Day subjects sat in a chair with a circular diffuse white light, $2.5 \mathrm{~cm}$ in diameter, placed on a table in front of the subject about $1 \mathrm{~m}$ distant from the eyes. For the Night subjects a similar stimulus-light was mounted near the foot of the bed at about the same aspect with respect to the eyes. The experimenter communicated directly with the Day subjects. The Night subjects slept and were tested in a light- and sound-controlled room with communication via a telephone and speaker system.

\section{Time Intervals}

For the first and last (usually eighth) test sessions the given time intervals were the whole-digit numbers of seconds from 1 to 10 . An estimation, production, and reproduction trial occurred for each time interval according to a random schedule, except that the same method or the same time interval was not allowed to occur more than twice in succession.

For the second to the seventh test sessions (following awakenings for the Night subjects), three time intervals for each of the three methods were presented. The intervals were arranged in sets of 1-4-7, 2-5-8, and 3-6-9 sec and combined with the three methods so that each interval occurred once per session and the interval $\times$ method combinations occurred approximately evenly distributed over the six sessions. Within sessions, the nine-trial sequence was randomly determined but without allowing the same method to occur more than twice in succession.

\section{Test Sessions}

At the first session, the subjects were instructed in the task and given as many practice trials as necessary to achieve proficient performance. After a brief pause, the 30 trials of the first session were presented. The Night subjects were then allowed to go to sleep. The Day subjects left the laboratory with the request to return $1 \mathrm{~h}$ later.

During the night, time judgments were obtained after 10-min periods of stage 1 -REM, 2 , or 4 . We attempted to obtain two different test sessions preceded by at least $10 \mathrm{~min}$ of each EEG stage for each subject. At least $30 \mathrm{~min}$ was allowed to elapse between successive test sessions. The EEG was monitored visually, and when an acceptable 10-min period occurred, the experimenter awakened the subject by calling his name through the loudspeaker. The subject answered on the telephone indicating that he was ready for the time-judgment trials. When the nine trials were completed, he was allowed to go back to sleep. At the final awakening, the subject was given 30 time-judgment trials, as at the first session, and allowed to leave the laboratory.

For the Night subjects, Session 1 occurred between 2330 and 0030 and Session 8 between 0630 and 0830 , with approximately 40-75 min intervening between successive sessions. The average time of sleep onset was 0030 (range 2345-0120). Half of the Day subjects began at 0800 and returned at hourly intervals, commencing Session 8 at 1500 . The other half began at 0830 , returning at hourly intervals on the half-hour and commencing Session 8 at 1530. Oral temperatures were taken for the Day subjects prior to beginning the time-judgment trials at each session.

\section{RESULTS}

The distributions of EEG stages prior to the awakenings are given in Table 1 . One instance of each stage was obtained for all 20 Night subjects. Two of each were obtained for 14 subjects. A second occasion of stage 1-REM was not realized for three subjects, and a second occasion of stage 4 was not realized for another three subjects. These six subjects had a total of seven sessions, and the analyses as a 
Table 1

Number of Experimental Awakenings Following Each EEG Stage

\begin{tabular}{lrrrrrr}
\hline & \multicolumn{6}{c}{ Session } \\
\cline { 2 - 7 } Stage & 2 & 3 & 4 & 5 & 6 & 7 \\
\hline 1 -REM & 0 & 0 & 2 & 12 & 11 & 12 \\
2 & 4 & 12 & 7 & 7 & 8 & 2 \\
4 & 16 & 8 & 11 & 1 & 1 & 0 \\
\hline
\end{tabular}

function of sessions are based on seven sessions for the Night subjects. The time judgments were completed within about $3 \mathrm{~min}$ after the awakening signal.

A trend analysis was performed on the time judgments as a function of the 10 presented time intervals for the first and last sessions with no resulting significant departures from linearity. For the three values in a single function for the other sessions, the deviation of the response for the middle time interval was calculated from the straight line connecting the responses to the shortest and longest time intervals. These deviations did not differ significantly from zero nor vary significantly as a function of sessions. The slopes and intercepts of the best-fitting straight lines to the response $\times$ time functions can therefore be considered to be good representations of the data.

The slope and the intercept were computed for each function for each session for each subject. The response values were weighted inversely as their variances in the normal equations of the computation, providing more reliable coefficients than were yielded by the unweighted coefficients (Williams, 1959, pp. 19, 67). For estimation and reproduction, the slopes and the $\mathrm{Y}$-intercepts were the scores used in the following analyses. For production, the reciprocal of the slope and the $\mathrm{X}$-intercept were used.

The average slope-scores for Production and Estimation as a function of testing session are shown in Figure 1. A trend analysis for the Night subjects indicated a main effect in the quadratic component, $F(1,19)=32.31, p<.001$, and an overall difference in level for Production and Estimation, $F(1,19)=$ $10.67, \mathrm{p}<.005$. A similar analysis for the Day subjects showed a main effect in the quadratic component, $F(1,19)=5.93, p<.025$, and a Production by Estimation interaction in the quadratic component, $F(1,19)=8.28, p<.01$, but no difference between Production and Estimation in overall level. No linear component was significant, but the only function in Figure 1 not exhibiting a significant curvilinear trend was the Production function for the Day subjects.

The intercepts for Production did not vary systematically over sessions for either the Day or the Night subjects. The overall mean Production $(X-)$ intercepts were $.16(\mathrm{SD}=.30)$ for the Day subjects and $.21(\mathrm{SD}=.36)$ for the Night subjects.
The Estimation intercepts are shown in Figure 2. The quadratic component of the trend was significant for both the Day subjects, $F(1,19)=7.06$, $\mathrm{p}<.025$, and the Night subjects, $\mathrm{F}(1,19)=4.91$, $\mathrm{p}<.05$. Clearly, the directions of the trends for the Estimation intercepts (Figure 2) were opposite to those for the Estimation slopes (Figure 1). In particular, the lowest slopes and highest intercepts occurred for Awakenings 2 and 3 (Test Sessions 3 and 4 for the Night subjects).

The curvilinear shape of the trends obtained is itself an indication that the results were not dependent on EEG stage. Session 2 was predominantly

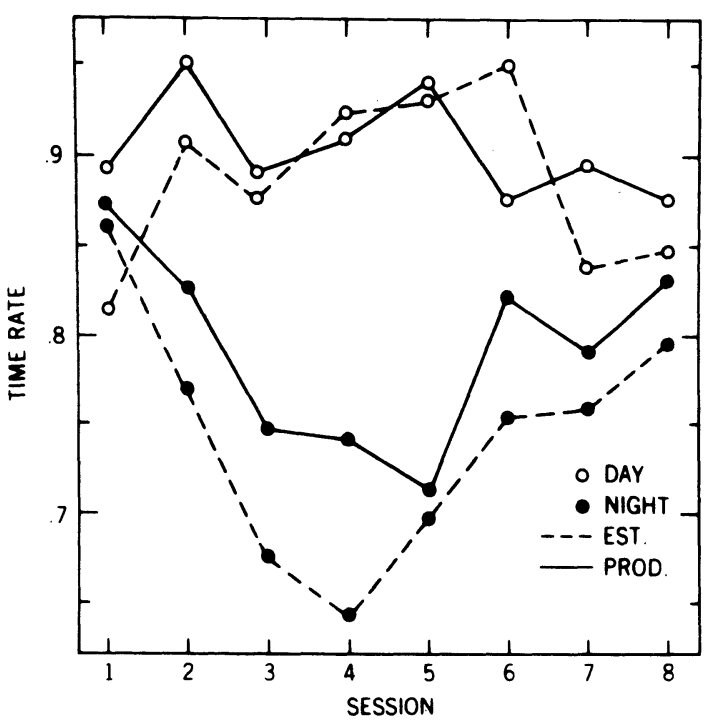

Figure 1. Subjective time rate as a function of test session for Day and Night subjects (rate = slope for time estimation, reciprocal of slope for time production).

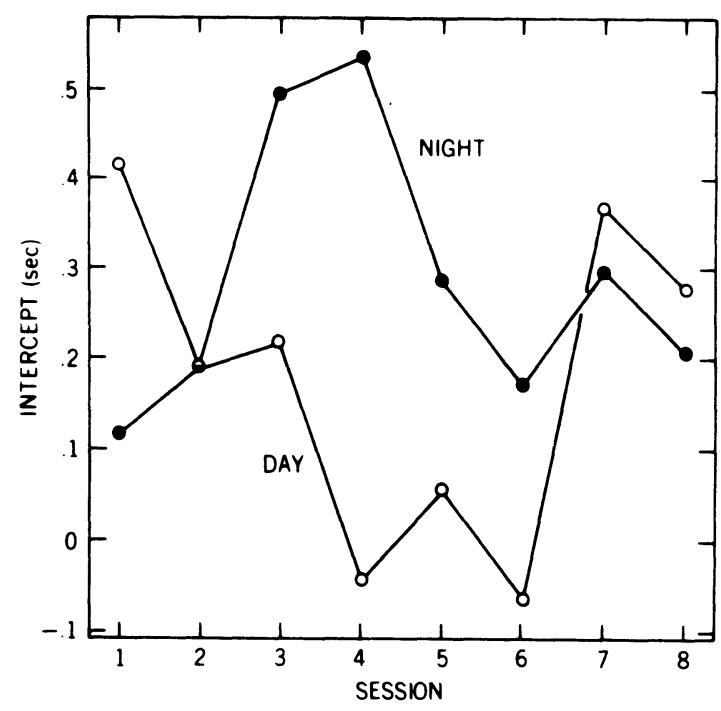

Figure 2. Time estimation intercept as a function of test session for Day and Night subjects. 
preceded by stage 4 with no stage 1-REM, while Session 7 was predominantly preceded by stage 1 -REM with no stage 4 (see Table 1). Yet the time judgment results were generally similar for Sessions 2 and 7. If the session trends were due to the distribution of EEG stages, there should be a significant effect with EEG stage as the independent variable, since the session trends were significant. On the other hand, if the session trend and EEG-stage effects were different, there should be a significant effect in the deviations of the time values for EEG stages from the session trend. Hence, both the scores and the deviations of the scores from the quadratic session trends were analyzed as a function of EEG stage preceding the awakenings.

None of the slope scores or deviations provided a statistically significant result due to EEG stage. For Estimation, the intercepts differed, $F(2,38)=5.61$, $\mathrm{p}<.01$, and the intercept deviations also differed $\mathrm{F}(2,38)=4.38, \mathrm{p}<.025$. Following an awakening from stage 4, the average Estimation intercept was close to what would be expected from the general session trend; following an awakening from stage 2 it tended to be relatively higher; following an awakening from stage 1-REM, relatively lower (Table 2). A similar effect occurred in the intercepts for the Production functions but was not statistically significant.

The oral temperature readings obtained on the Day subjects rose from an average of $36.24^{\circ} \mathrm{C}$ in the morning to $36.76^{\circ} \mathrm{C}$, declined slightly to $36.66^{\circ} \mathrm{C}$ over noontime, and then rose again to $36.83^{\circ} \mathrm{C}$ in the afternoon. The linear and cubic components of the trend were both highly significant, $F(1,19)=$ 46.35 and 47.28 , respectively, $p<.001$. This trend was thus different from the trends for the time judgments.

Neither the slopes nor the intercepts of the Reproduction functions varied systematically in relation to sessions or any other variable. The overall averages were: for the Day subjects, slope $=.98(\mathrm{SD}=.08)$, intercept $=-.21(\mathrm{SD}=.33)$; for the Night subjects, slope $=.99(\mathrm{SD}=.14)$, intercept $=-.14(\mathrm{SD}=.45)$.

\section{DISCUSSION}

Whatever the subjective time rate might be, so long as it is the same for both parts of a reproduction trial, the slope of that function should be 1 . The reproduction slopes showed no systematic deviation from a slope of 1 , hence there was no systematic impairment in discriminating the time intervals employed or in the operation of holding the response switch closed for an interval equivalent to a presented time interval.

We take the difference in rates for estimation and production as indicating an inconsistency in respond-
Table 2

Average Estimation Intercepts (Seconds) Following EEG Awakenings and Deviations from General Quadratic Session Trend $(N=20)$

\begin{tabular}{lccr}
\hline & \multicolumn{3}{c}{ EEG Stage } \\
\cline { 2 - 4 } & 1-REM & 2 & 4 \\
\hline Mean & .15 & .52 & .29 \\
Session Trend & .30 & .35 & .34 \\
Deviation & -.15 & .17 & -.05 \\
SD & .49 & .42 & .28 \\
\hline
\end{tabular}

ing. Since the intercepts varied systematically for estimation but not for production, we infer that the trend in the slope scores for production was more nearly representative of the trend in subjective time rate. On this interpretation, time rate decreased to a minimum somewhat past the middle of the sleep period and increased toward morning with little change during the day.

All three time judgment tasks required the subject to press and release a switch. The most obvious difference therefore between estimation and the other two tasks was that estimation required the subject to report a verbal estimate of the presented interval. If verbal estimation is cognitively more demanding, the steeper decline to an earlier minimum for estimation is consistent with Snyder and Scott's (1972) hypothesis that cognitive functioning is more affected early in sleep. In any event, the lack of relationship between the slope values and the EEG stages preceding the awakenings suggests that there was no differential effect of sleep state on subjective time rate.

Since the slopes of the functions were not affected by the stage of sleep, the differential effect on the estimation intercepts indicates a greater constant response error for stage 2 than for stage 1-REM. The possibility of this kind of error is plausible enough. If, in counting off the seconds, subjects sometimes counted beyond the time the stimulus light went off, an increment would be added to the intercept. And less error for stage 1-REM is consistent with observations that performance following REM sleep is more like daytime performance than performance following non-REM sleep (Lavie, 1974; Snyder \& Scott, 1972). But it is puzzling why such errors were not as great, or greater, following stage 4 (deep) sleep. Differential sleep effects on reaction time have been obtained both sooner (Goodenough, Lewis, Shapiro, Jaret, \& Sleser, 1965) and later (Wilkinson \& Stretton, 1971) from the time of awakening than the present time judgment trials. It appears likely, therefore, that carryover effects from the sleep condition were present at the time of testing.

The minima of the slopes of the time judgment functions occurred earlier in the sleep period than would be expected on the basis of decline in body 
temperature (Aschoff, Fatranska, Gerecke, \& Giedke, 1974), and the daytime values did not correspond to the variation in body temperature. Adam, Castro, and Clark (1974) and Adam, Rosner, Hosick, and Clark (1971) found that inhalation of anesthetic gases decreased time rate as measured by the slopes of the time judgment functions, but the changes in time rate were not accompanied by concomitant changes in alpha rhythm, respiratory rate, heart rate, or body temperature. Repetition of these time judgment tasks tends to produce a slowing in time rate followed by a return to the initial rate (Carlson \& Feinberg, 1970). This tendency may have acted in the same direction as the trend over the first half of the night and counteracted the effect of temperature during the latter part of the night and during the day. If so, however, one must conclude that the effect of repetition is as important as the effect of temperature in determining time rate.

The present results agree with those of Aschoff et al. (1972) and Wever (1972), indicating an overall circadian variation in internal clock rate which generally parallels the sleep and temperature cycles but is not specifically dependent on normal variations in body temperature nor on the stages of sleep identified by the EEG. An increased tendency toward indiscriminate responding appears to occur upon awakening from sleep, and it is this aspect of performance rather than the subjective time rate which may vary as a function of the preceding EEG stage of sleep.

\section{REFERENCES}

Adam. N.. Castro, A. D.. \& Clatr., D. L. Production, estimation, and reproduction of time intervals during inhalation of a general anesthetic in man. Journa! of Experimental Psycholog!' 1974, 102, 609-614.

Adam. N.. Rosner. B. S., Hosick. E. C., \& Clark, D. L. Effect of anesthetic drugs on time production and alpha rhythm. Perception \& Psychophysics, 1971, 10, 133-136.

Aschoff, J.. Fatranska, M., Gerecke, U., \& Giedke, H. Twenty-four-hour rhythms of rectal temperature in humans: Effects of sleep-interruptions and of test-sessions. Pflügers Archiv, 1974. 346. 215-222.
Aschoff. J.. Giedke. H.. Poppel, E., \& Wever, R. The influence of sleep-interruption and sleep-deprivation on circadian rhythms in human performance. In W. P. Colquhoun (Ed.). Aspects of human efficiency: Diurnal rhythm and loss of sleep. London: English Universities Press, 1972. Pp. 135-149.

Carlson. V. R. \& Feinberg. I. Individual variations in time judgment and the concept of an internal clock. Journal of Experi. mental Psychology: 1968, 77, 631-640.

Carlson. V. R.. \& Feinberg. I. Time judgment as a function of method, practice, and sex. Joumal of Experimental Psychology', 1970. 85, 171-180.

Feinberg. I., Koresko. R. L., \& Heller, N. EEG sleep patterns as a function of normal and pathological aging in man. Journal of Psichiatric Research. 1967. 5. 107-144.

Goodenough, D. R., Lewis, H. B.. Shapiro, A., Jaret, L., \& Sleser. I. Dream reporting following abrupt and gradual awakenings from different types of sleep. Journal of Personality and Social Psychology. 1965, 2, 170-179.

LAVIE. P. Differential effects of REM and non-REM awakenings on the spiral aftereffect. Physiological Psychology, 1974. 2. 107-108.

Mangold. R.. Sokoloff, L., Conner, E., Kleinerman, J., Therman. P. G., \& Kety. S. The effects of sleep and lack of sleep on the cerebral circulation and metabolism of normal young men. Journal of Clinical Investigation, 1955. 34, 1092-1100.

Reibich, M., Isaacs, G., Evarts, E., \& Kety, S. Regional cerebral blood flow during REM and slow wave sleep. Transactions of the American Neurological Association, 1967, 92, 70-74.

SNYDER. F., \& SCOTT. J. The psychophysiology of sleep. In N. S Greentield. \& R. A. Sternbach (Eds.), Handbook of psychophysiology. New York: Holt. Rinehart, \& Winston, 1972. Pp. 645-708.

WeVer, R. Circadian rhythms in human performance. Paper presented at NATO symposium on drugs, sleep, and performance. Aviemore. Scotland, 1972. (Described by Lavie, P. Rhythms in human performance, JSAS Catalog, 1974, 4, 39, Ms. No. 610. pp. 46-47.)

Wilkinson, R. T., \& Stretton, M. Performance after awakening at different times of night. Psychonomic Science, 1971, 23, 283-285.

Williams. E. J. Regression analysis. New York: Wiley, 1959.

\section{NOTES}

1. Random variation around a fixed population function will produce a negative correlation between the calculated slopes and intercepts. We are here concerned with systematic variation in the population parameters.

\section{(Received for publication March 2, 1976;} accepted March 29. 1976.) 